\title{
OBSERVAÇÕES SOBRE CALAZAR EM JACOBINA, BAHIA. VI - INVESTIGAÇÕES SOBRE RESERVATÓRIOS SILVESTRES E COMENSAIS.
}

\author{
I. A. Sherlock ${ }^{1}$, J.C. Miranda ${ }^{1}$, M. Sadigursky ${ }^{1}$ e G. Grimaldi Jr. ${ }^{2}$
}

\begin{abstract}
Durante os anos de 1982 a 1986, a investigação sobre mamiferos comensais $e$ silvestres, da periferia da cidade de Jacobina, Bahia, mostrou, ao lado do escasso número de exemplares, uma reduzida variedade especifica dessa fauna. Capturou-se apenas 11 espécies, entre as quais, predominou o Didelphis albiventris, que abrangeu 44\% dos 213 espécimens capturados. Entre os 193 com exames já concluídos, 84 eram exemplares de D. albiventris $e 2$ estavam infectados pela Leishmania donovani senso lato, 1 por $\mathrm{L}$. mexicana amazonensis, 1 por $\mathbf{L}$. braziliensis, subespécie e 3 por Trypanosoma cruzi Também foram observadas formas suspeitas de serem amastigotas de leishmanics, nos esfregaços de órgãos de 3 exemplares de Dasyprocta aguti, 1 Cercomys cunicularius - $e$ 1 Oryzomys eliurus. $O$ restante dos exemplares, inclusive 14 de Lycalopex vetulus, estava negativo para flagelados.

Apesar de reforçado por outros indicadores epidemiológicos, como a predominância especifica, a freqüência domiciliar, a atratividade para a vetora Lutzomyia longipalpis, e a concomitância com casos humanos nos mesmos locais, o indice de $2,3 \%$ de infecção natural do Didelphis albiventris, não autoriza a conclusão definitiva de sero marsupial o mais importante reservatório natural da leishmaniose visceral em Jacobina.
\end{abstract}

Palavras Chaves: Leishmaniose visceral americana. Reservatórios silvestres. Didelphis albiventris. Leishmania donovani. Leishmania mexicana amazonensis. Leishmania brasiliensis subespécie.

Com exceção de um trabalho dos próprios autores do presente ${ }^{9}$, nada mais existe publicado sobre reservatórios extradomésticos da leishmaniose visceral americana em Jacobina, apesar de ser esse um dos mais antigos focos de calazar do Brasil.

Aqui apresentamos alguns resultados de investigações recentes (de 1982 a 1986) que fizemos em Jacobina, Bahia, sobre reservatórios comensais e silvestres de leishmaniose visceral, dando continuidade à publicação da série de observações sobre ecologia e epidemiologia da leishmaniose visceral nesse foco endêmico, que vinham sendo feitas desde 1959 pelo primeiro autor 456789 .

\section{MATERIAL E MÉTODOS}

As zonas periféricas da cidade de Jacobina foram hipoteticamente divididas em sete subáreas, seguindo o critério de Teixeira ${ }^{10}$ e Badaró ${ }^{1}$ que também faziam simultaneamente observações sobre os aspectos clínicos e epidemiológicos do calazar na mesma área. A subárea 3, Grotinha, onde mais recentemente

1 Centro de Pesquisas Gonçalo Moniz - FIORUZ/UFBa Salvador, Bahia.

2 Instituto Oswaldo Cruz FIOCRUZ - Rio de Janeiro.

Financiado pelo International Development Research Centre, Canadá.

Recebido para publicação em 9/3/87. vinha ocorrendo maior número de casos humanos da doença, foi a escolhida para servir de área de amostragem para o nosso trabalho. Grotinha corresponde a um pequeno vale, onde existem três tuas de casas desordenadamente localizadas. Fica contínua à área que possui os últimos resquícios de vegetação, da periferia da cidade, representados por matas de formação secundária. No periodo em que realizamos as observações aqui relatadas, a leishmaniose visceral apresentou-se com transmissão ativa, apesar de que nos anos iniciais, apenas poucos casos tenham sido diagnosticados. No final do periodo de observações, ocorreu um surto epidêmico, quando foram registrados mais de 50 casos humanos. De 1980 a 1984, segundo Badaró ${ }^{1}$, a incidência média global de casos foi de 13,7/1.000 crianças nessa subárea de Grotinha.

Todo mês, durante uma semana, colocávamos armadilhas de arame tipo alçapão para a coleta de pequenos animais silvestres e algumas para animais maiores, em pontos que julgávamos estratégicos. As armadilhas eram colocadas pela manhã e à noite, quando eram também feitas inspeções para ver se tinham capturado algum animal, e para reposição das iscas que já tinham sido consumidas. Usamos como iscas frutas, milho, queijo, semente de abóbora, etc.; a isca de abacaxi era a que dava melhores resultados para a captura de marsupiais e a de semente de abóbora, para roedores. Além dos animais que coletamos, adqui- 
rimos, dos caçadores locais, os exemplares de raposas, cutias e tatus referidos neste trabalho. É muito difícil a captura de raposas e por isso jamais coletamos um só exemplar em nossas armadilhas.

Todos os animais foram submetidos a xenodiagnósticos com triatomídeos e flebotomíneos. Após sacrificados sob anestesia geral com éter, clorofórmio, tionembutal ou pentobarbital, era feita a necrópsia e retirava-se de cada animal um fragmento de figado, baço, pele e uma amostra de sangue do coração. Esta última era imediatamente examinada para a pesquisa de tripanossomas. As amostras de todo o outro material eram utilizadas para esfregaços em lâminas que eram corados pelo Giemsa, para o semeio em meios de cultura para flagelados (NNN, LIT, etc.), inclusive o sangue, e para o estudo histopatológico. Os esfregaços em lâminas foram examinados ao microscópio ótico, para a pesquisa de amastigotas. Além desses exames as amostras de fígado e baço eram maceradas e inoculadas intraperitonealmente em hamsteres. Estes animais. a não ser que se apresentassem doentes antes do tempo, eram examinados no periodo de 6 a 12 meses a contar da data da inoculação.

As espécies dos animais que foram coletadas eram comuns e já conhecidas, não tendo sido necessária uma classificação especial. Nós mesmos as identificamos, comparando-as com exemplares taxidermizados que tinham sido classificados por especialistas do Museu Nacional. A identificação das espécies de leishmânias foi feita nos laboratórios do Instituto Oswaldo Cruz, através de análise da densidade especifi- ca do DNA, anticorpos monoclonais e composição isoenzimática, assim como também em nossos laboratórios, pelo comportamento de infecção no hãmster $\mathrm{e}$ no tubo digestivo de L. longipalpis, crescimento em culturas e aspecto morfológico em esfregaços corados pelo Giemsa.

\section{RESULTADOS}

Foram montadas 3.353 armadilhas para a captura dos animais silvestres e comensais em Jacobina, durante os anosde 1982 a 1986, das quais, apenas 166 (5\%) conseguiram capturar 178 animais. Isso demonstrou, não a falta de eficácia das armadilhas, mas sim, o escasso número de animais silvestres existentes na periferia da cidade.

Além desses animais que capturamos e que foram representados, na quase totalidade, por marsupiais e roedores, foram adquiridos por compra dos caçadores locais, 8 cutias, 6 tatus, 7 preás e 14 łaposas.

Ao lado do reduzido número de espécimens, a variedade especifica da fauna era também muito pequena, tendo-se conseguido, incluindo-se os roedores doméstico, apenas 11 espécies de mamíferos, conforme apresentamos na Tabela 1.0 animal predominante foi o marsupial Didelphis albiventris, localmente chamado de "sariguê", que abrangeu $44 \%$ dos animais examinados da área. Em seguida, predominavam os roedores, com cerca de $39 \%$ de freqüência. A rapo-

Tabela 1 - Animais silvestres e comensais obtidos de 1982 a 1986, em Jacobina, Bahia, para a pesquisa de leishmânias.

Espécie de animal

$\begin{array}{ccc}\text { Capturados } & \text { Adquiridos } & \\ \text { em } & \text { dos } & \text { Total } \\ \text { Armadilhas } & \text { Habitantes } & \end{array}$

\begin{tabular}{lccr}
\hline Didelphis albiventris (Sariguê, gambá) & 94 & - & 94 \\
Dasyprocta aguti (Cutia) & - & 8 & 8 \\
Cavia porcellus (Preá) & - & 7 & 7 \\
Cercomys cunicularius (Rato-punurá) & 12 & - & 12 \\
Oryzomys eliurus & 8 & - & 8 \\
Orysomys subflavus (Rato-do-algodão) & 1 & - & 1 \\
Rattus rattus (Rato preto, guabiru) & 20 & - & 20 \\
Mus musculus (Camundongo doméstico) & 42 & - & 42 \\
Chiroptera (espécie?) (morcego) & 1 & - & 1 \\
Cabassous unicinctus & - & 6 & 6 \\
Lycalopex vetulus (Raposa)* & - & 14 & 14 \\
\hline
\end{tabular}

* Identificamos como Lycalopex vetulus por nos parecerem idênticos aos estudados por L.M. Deane ${ }^{2}$ no Ceará e assim determinados pelo Dr. João Moojen, do Museu Nacional. Os exemplares de Cerdocyon thous, capturados no Pará por R. Lainson ${ }^{3}$, parecem-nos maiores e mais escuros. 
sa Lycalopex vetulus apresentou-se com apenas 14 exemplares. Os roedores silvestres foram menos freqüentes que os domésticos.

Foram obtidos e examinados para leishmânias 213 animais (Tabela 1). Entre os 193 com exames já concluidos (Tabela 2), foram encontrados com formas suspeitas de serem amastigotas de leishmânias, esfregaços de baço e figado de 4 exemplares de Dasyprocta aguti e esfregaços de baços de 1 Cercomys cunicularius e 1 Oryzomys eliurus. Infelizmente, não foi possível o isolamento, nem a identificação específica dessas suspeitas leishmânias.

Tabela 2 - Animais silvestres e comensais obtidos de 1982 a 1986 em Jacobina, Bahia, encontrados naturalmente infectados por trypanosomatideos

\begin{tabular}{|c|c|c|c|c|c|c|}
\hline \multirow[b]{2}{*}{$\begin{array}{c}\text { Espécie } \\
\text { do } \\
\text { animal }\end{array}$} & \multirow[b]{2}{*}{ 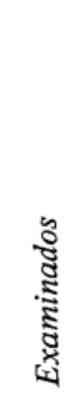 } & \multicolumn{3}{|c|}{ Com infecção por: } & \multirow[b]{2}{*}{ 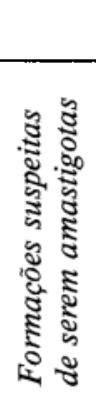 } & \multirow[b]{2}{*}{ 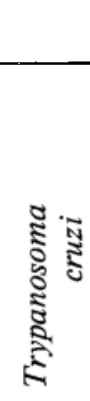 } \\
\hline & & 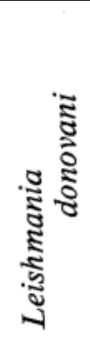 & 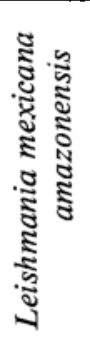 & 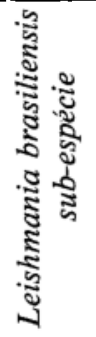 & & \\
\hline Didelphis albiventris & 84 & 2 & 1 & 1 & - & 3 \\
\hline Dasyprocta aguti & 7 & - & - & - & 4 & - \\
\hline Cavia porcellus & 6 & - & - & - & - & - \\
\hline Cercomys cunicularius & 12 & _- & - & - & 1 & - \\
\hline Oryzomys eliurus & 8 & - & - & - & 1 & \\
\hline Oryzomys subflavus & 1 & - & - & - & - & - \\
\hline Rattus rattus & 19 & - & - & - & - & - \\
\hline Mus musculus & 35 & - & - & - & _- & - \\
\hline Chiroptera (espécie?) & 1 & - & - & - & - & - \\
\hline Cabassous unicinctus & 6 & - & - & - & - & - \\
\hline Lycalopex vetulus & 14 & - & - & - & - & - \\
\hline Total & 193 & 2 & 1 & 1 & 6 & 3 \\
\hline
\end{tabular}

Entre os 94 exemplares de D. albiventris coletados, já têm resultados definitivos 84 e destes 4 estavam infectados com 3 espécies de leishmânias e $3 \mathrm{com}$ Trypanosoma cruzi, conforme mostramos na Tabela 2. Os exemplares infectados de $D$. albiventris, de acordo com as espécies de leishmânias, e pelo $T$. cruzi, foram: com Leishmania donavani lato sensu 2 exemplares nas inoculaçōes de baços em hamsteres; Leishmania mexicana amazonensis 1 exemplar infectado na hemocultura; Leishmania braziliensis subespécie 1 exemplar infectado em inoculações distintas de fígado e baço, em hamsteres; Trypanosoma cruzi em 3 exemplares infectados, sendo 2 através de hemoculturas e 1 através de xenodiagnóstico com triatomíneos.

Na Tabela 3, observa-se que os exemplares de D. albiventris foram coletados durante todos os meses do ano, havendo, contudo, um maior número de animais nos meses de junho, julho e agosto. Essa variação estava diretamente correlacionada com a den-
Tabela 3 - Variação mensal de Didelphis albiventris em Jacobina, Bahia (1982-1986).

\begin{tabular}{|c|c|c|c|}
\hline Mês & $\begin{array}{l}\text { No armadilhas } \\
\text { colocadas }\end{array}$ & $\begin{array}{l}\text { No } D \text {. albiven- } 1 \\
\text { tris capturados }\end{array}$ & $\begin{array}{l}\text { ice armadilhas } \\
\text { positivas }\end{array}$ \\
\hline Janeiro & 214 & 4 & 1,9 \\
\hline Fevereiro & 154 & 2 & 1,3 \\
\hline Março & 233 & 2 & 0,9 \\
\hline Abril & 299 & 7 & 2,3 \\
\hline Maio & 334 & 6 & 1,8 \\
\hline Junho & 342 & 4 & 1,2 \\
\hline Julho & 353 & 17 & 4,8 \\
\hline Agosto & 408 & 14 & 3,4 \\
\hline Setembro & 265 & 2 & 0,8 \\
\hline Outubro & 280 & 8 & 2,9 \\
\hline Novembro & 252 & 6 & 2,4 \\
\hline Dezembro & 218 & 7 & 2,8 \\
\hline Total & 3.353 & 79 & 2,4 \\
\hline
\end{tabular}

* Não incluidos 15 fillhotes capturados com as mães. 
sidade de Lutzomyia longipalpis e com os casos humanos de leishmaniose visceral, conforme pode ser observado em trabalhos já publicados 7810 .

\section{DISCUSSÃO}

Devido, provavelmente, à exterminação indiscriminada feita pelo homem, a fauna de mamíferos silvestres e comensais, que ainda existe na periferia de $\mathrm{Ja}$ cobina, restringe-se a poucas espécies. Entre as remanescentes, destaca-se o marsupial Didelphis albiventris, ali encontrado, pela primeira vez, infectado pela Leishmania donovani lato sensu, com o indice de 2,3\% de infecção natural. Esse mamífero, foi também encontrado em Jacobina, naturalmente infectado pelas seguintes outras espécies de leishmânias: $L$. mexicana amazonensis $(1,1 \%), L$. braziliensis subespécie (1,1\%), e pelo Trypanosoma cruzi $(3,5 \%)$.

As leishmanioses cutâneas são raras em Jacobina e a doença de Chagas não existe de modo autóctone nessa localidade. A falta de ocorrência dessa tripanosomose humana é explicada pela inexistência de triatomíneos domiciliados e, por isso, somente poucos casos humanos, não autóctones, têm sido registrados na cidade 110

Lutzomyia flaviscutellata, que é a principal vetora de $L$. m. amazonensis, não foi assinalada para Jacobina. Entretanto, outra espécie do subgênero Nyssomyia, a Lutzomyia (N.) whitmani, já foi encontrada na área, podendo ser uma das responsáveis pela transmissão desses tipos de leishmânias. A Lutzomyia longipalpis, a vetora principal da $L$. donovani no Brasil, é bem atraída para sugar o $D$. albiventris, tanto no campo como no laboratório ${ }^{9}$, sendo também uma vetora potencial das outras espécies de leishmânias mencionadas.

Os dados foram obtidos durante os meses mais chuvosos do ano, e quando o marsupial com mais freqüência procura o domicilio humano, em busca de alimento, o qual torna-se, provavelmente, mais escasso, nos ecótopos silvestres. No ambiente domiciliar, além de restos de comida, o marsupial se alimenta de ovos, galinhas, pintos, que rouba nos abrigos desses animais domésticos.

Além de sua infecção natural, os seguintes fatores corroboraram para incriminar, epidemilogicamente, o $D$. albiventris, como um reservatório primário de $L$. donovani em Jacobina: a variação estacional da atividade domiciliar do mamifero que é correlacionada com a variação estacional da população do vetor e com a prevalência da doença humana e da canina; encontra-se, concomitantemente, em grutas de pedras, tanto $L$. longipalpis como o marsupial; esse mamifero freqüenta o peridomicílio, vindo do ambiente silvestre. Os dois exemplares, que foram encontrados naturalmente infectados, foram capturados numa casa vizinha a uma outra onde, simultaneamente, encontramos um cão doente. Essa casa, por sua vez, era muito próxima a uma terceira casa onde se diagnosticou, ao mesmo tempo, um caso humano de calazar; infecta-se facilmente $\operatorname{com} L$. donovani e não sofre danos aparentes provocados pelo parasito. $\mathrm{O}$ estudo dos exemplares, naturalmente infectados, e também o de um exemplar infectado, experimentalmente, com a mesma cepa de leishmânia, mostrou discretas alterações histopatológicas, apesar de ter sido usada elevada dose de parasitos no inóculo da observação experimental.

$O D$. albiventris é um animal americano e sua infecção por uma leishmânia que não the provoca danos acentuados, parece indicar uma antiga associação parasito-hospedeiro no continente americano. Assim, poder-se-ia explicar a origem americana de uma espécie de leishmânia viscerotrópica que já foi denominada de Leishmania chagasi Cunha \& Chagas, 1937. O marsupial representaria um dos elos de conexão das cadeias de transmissão doméstica e silvestre.

Apesar dessas fortes evidências, ainda não se pode afastar a possibilidade de ter sido o marsupial infectado a partir do homem, de cães ou de roedores, infectados pela $L$. donovani, que tenham sido introduzidos na área.

Também, em vista do pequeno número de exemplares examinados de outros mamiferos, incluindo-se raposas e roedores, não fica excluída a possibilidade de que esses tenham importantes papéis como reservatórios primários da leishmaniose visceral americana. $O$ baixo índice de infecção natural de $D$. albiventris pela $L$. donovani $(2,3 \%)$, apesar de sugerir, não autoriza a fazer-se uma conclusão definitiva sobre o assunto. Isto exige a realização de mais investigações para o esclarecimento de quais são os mais importantes reservatórios naturais de leishmaniose visceral no continente americano.

\section{AGRADECIMENTOS}

Agradecemos aos nossos técnicos auxiliares, a seguir relacionados, a colaboração que nos deram tanto nos trabalhos de campo como no laboratório: Antônio Carlos Santos, Jorge Lessa Tolentino, Ronaldo Pereira Lima e Raimundo José Ferreira.

\section{SUMMARY}

From 1982 to 1986, investigations on the natural infection with leishmanias of the sylvatic and commensal mammals were carried out around the city of Jacobina, Bahia, one of the oldest endemic foci of visceral leishmaniasis in Brazil. 
The species of Marsupialia Didelphis albiventris predominated, with the rate of $44 \%$, over the total of 213 specimens belonging to only 11 different species of mammals collected there. Among the 84 specimens of $\mathrm{D}$. albiventris examined, $2(2.3 \%)$ were infected with Leishmania donovani sensu lato; 1 with L. mexicana amazonensis, 1 with L. braziliensis sub species, and 3 with Trypanosoma cruzi. Also, amastigote suspected bodies were seen in the smears of spleens and livers of 3 Dasyprocta aguti, 1 Cercomys cunnicularius and 1 Oryzomys sp.

Although strengthened by some epidemiological evidences, such as specific predominancy, peridomestic and domestic occurrence, attractiveness for the vector Lutzomyia longipalpis and the concomitancy with human cases of visceral leishmaniasis at the same place, the low rate of the natural infection of $\mathrm{D}$. marsupialis still do not allow a definitive conclusion that the oppossum is a primary and the most important reservoir of visceral leishmaniasis in Jacobina.

\section{REFERÊNCIAS BIBLIOGRÁFICAS}

1. Bađaró RJ S. Epidemiologia da leismaniose visceral. Estudo prospectivo da infecção e doença na área endêmica de Jacobina-Bahia (1980-1984). Tese Mestrado. Universidade Federal da Bahia, 1985.

2. Deane LM. Leishmaniose visceral no Brasil. Estudos sobre reservatórios e transmissores realizados no Estado do Ceará. Servico Nacional de Educação Sanitária, Rio de Janeiro, 1956.
3. Lainson R. Our present knowledge of the ecology and control of Leishmaniasis in the Amazon Region of Brazil. Revista da Sociedade Brasileira de Medicina Tropical 18:47-56, 1985.

4. Sherlock IA. Observações sobre calazar em Jacobina, Bahia. I - Histórico e dados preliminares. Revista Brasileira de Malariologia e Doenças Tropicais 21:523-534, 1969.

4. Sherlock IA, Almeida SP. Observações sobre calazar em Jacobina, Bahia. II - Leishmaniose canina. Revista Brasileira de Malariologia e Doenças Tropicais 21:535540, 1969.

6. Sherlock IA, Almeida SP. Observaçōes sobre calazar em Jacobina, Bahia. V-Resultados de medidas profiláticas. Revista Brasileira de Malariologia e Doenças Tropicais 22:715-728, 1970.

7. Sherlock IA, Guitton N. Observações sobre calazar em Jacobina, Bahia. III - Alguns dados sobre o Phlebotomus longipalpis o principal transmissor. Revista Brasileira de Malariologia e Doenças Tropicais 21:541-548, 1969.

8. Sherlock IA, Guitton N. Observações sobre calazar em Jacobina, Bahia. IV - Variação horária e estacional do Phlebotomus longipalpis. Revista Brasileira de Malariologia e Doenças Tropicais 21:715-728, 1969.

9. Sherlock IA, Miranda JC, Sadigursky M, Grimaldi Jr. G. Natural infection of the opossum Didelphis albiventris (Marsupialia Didelphidae) with Leishmania donovani in Brazil. Memórias do Instituto Oswaldo Cruz 79:461-470, 1984.

10. Teixeira R. Experièncias vividas com a leishmaniose visceral 1954/1980 (Aspectos epidemiológicos, sorológicos e evolutivos). Tese. Universidade Federal da Bahia, 1980. 